\section{Temperature- and excitation intensity-dependent photoluminescence in TIInSeS single crystals}

To cite this article: N M Gasanly et al 2002 J. Phys.: Condens. Matter 1413685

View the article online for updates and enhancements.

\section{Related content}

- $\frac{\text { Radiative donor-acceptor pair }}{\text { recombination in TIInS }}$ singlecrystals
A Aydinli, N M Gasanly, I Yilmaz et al.
- $\frac{\text { Low-temperature visible }}{\text { photoluminescence spectra ofTI2GalnSe4 }}$
$\frac{\text { layered crystals }}{\text { B Abay, H Efeoglu, Y K Yogurtçu et al. }}$
- Visible photoluminescence from chain
$\frac{\text { TI4In3GaSe8 semiconductor }}{\text { N M Gasanly and K Goksen }}$

\section{Recent citations}
Phase diagram and specific band gap features of novel TIGaSe $2: \mathrm{Zn}+2(\mathrm{Cd}+2$ $\mathrm{Ha}+2$ ) crystals
G. Makhnovets et al
Structural and photoluminescence properties of Si-based nanosheet bundles rooted on Si substrates Peiling Yuan et al
Thermal transfer and interaction mechanisms of localized excitons in families of InAs quantum dashes grown on $\ln \mathrm{P}(001)$ vicinal substrate emitting near $1.55 \mathrm{~m}$ wavelength Fatiha Besahraoui et al




\title{
Temperature- and excitation intensity-dependent photoluminescence in TIInSeS single crystals
}

\author{
N M Gasanly ${ }^{1,3}$, A Aydinli ${ }^{2}$ and N S Yuksek ${ }^{1}$ \\ ${ }^{1}$ Physics Department, Middle East Technical University, 06531 Ankara, Turkey \\ 2 Physics Department, Bilkent University, 06533 Ankara, Turkey \\ E-mail: nizami@metu.edu.tr (N M Gasanly)
}

Received 18 September 2002

Published 29 November 2002

Online at stacks.iop.org/JPhysCM/14/13685

\begin{abstract}
Photoluminescence (PL) spectra of TlInSeS layered single crystals were investigated in the wavelength region $460-800 \mathrm{~nm}$ and in the temperature range 10-65 K. We observed one wide PL band centred at $584 \mathrm{~nm}(2.122 \mathrm{eV})$ at $T=10 \mathrm{~K}$ and an excitation intensity of $7.5 \mathrm{~W} \mathrm{~cm}^{-2}$. We have also studied the variation of the PL intensity versus excitation laser intensity in the range from 0.023 to $7.5 \mathrm{~W} \mathrm{~cm}^{-2}$. The red shift of this band with increasing temperature and blue shift with increasing laser excitation intensity was observed. The PL was found to be due to radiative transitions from the moderately deep donor level located at $0.243 \mathrm{eV}$ below the bottom of the conduction band to the shallow acceptor level at $0.023 \mathrm{eV}$ located above the top of the valence band. The proposed energy-level diagram permits us to interpret the recombination processes in TlInSeS layered single crystals.
\end{abstract}

\section{Introduction}

Quaternary thallium chalcogenide TlInSeS is formed from the $\mathrm{TlInS}_{2}-\mathrm{TlInSe}_{2}$ system and has a direct band gap of $2.328 \mathrm{eV}$ at $T=10 \mathrm{~K}$ [1]. In view of possible optoelectronic device applications in the visible region, a great deal of attention has been devoted to the study of the optical and electrical properties of thallium chalcogenides [2-5]. Long-wave optical phonons in TlInSeS crystals were investigated by infrared reflection and Raman scattering experiments $[6,7]$. Photoluminescence (PL) spectroscopy is a very suitable and widely used technique to study optical transitions in semiconductors. However, no PL study of TlInSeS crystals has been reported so far. Recently, we have measured the PL spectra of TlInS $\mathrm{S}_{2}$ in the 11.5-100 K temperature range and observed broad emission bands, which we have attributed to donor-acceptor pair recombinations [8].

\footnotetext{
3 Author to whom any correspondence should be addressed.
} 
In this paper, we present the results of a systematic experimental analysis of the PL of TlInSeS single crystals in the wavelength region 460-800 nm and in the 10-65 K temperature range. The PL spectra and their temperature and excitation intensity dependencies were studied in detail in order to propose a model for the recombination process of photoexcited carriers and a scheme for the impurity levels.

\section{Experimental details}

TIInSeS polycrystals were synthesized from particular high-purity elements (at least 99.999\%) taken in stoichiometric proportions. Single crystals of TIInSeS were grown from the melt by the Bridgman technique. The x-ray diffraction patterns show that these crystals have monoclinic structure with the lattice parameters $a=1.111, b=1.062$ and $c=1.592 \mathrm{~nm}$ [9]. Crystals suitable for measurements were obtained by easy cleavage along the (001) plane perpendicular to the optical $c$ axis. In the PL measurements, typical sizes of the samples were $6 \times 3 \times 2 \mathrm{~mm}^{3}$. All the samples studied were p-type as determined by the hot probe method. The surface of the sample was irradiated along the $c$ axis by an argon-ion laser using the $457.9 \mathrm{~nm}$ line. The emission was collected in the back-scattering geometry from the face of the crystal. A 'CTI-Cryogenics M-22' closed-cycle helium cryostat was used to cool the crystals from room temperature down to $10 \mathrm{~K}$. The temperature was controlled to within an accuracy of $\pm 0.5 \mathrm{~K}$. The PL spectra in the wavelength range 460-800 nm were analysed using a U-1000 'Jobin Yvon' double grating spectrometer and a cooled GaAs photomultiplier supplied with the necessary photon counting electronics. The PL spectra have been corrected for the spectral response of the optical apparatus. A set of neutral-density filters changed the intensity of the laser beam in the range from 0.023 to $7.5 \mathrm{~W} \mathrm{~cm}^{-2}$.

\section{Results and discussion}

Figure 1 shows the PL spectra of TlInSeS single crystals measured in the wavelength region $500-700 \mathrm{~nm}$ and in the $10-65 \mathrm{~K}$ temperature range at a constant excitation laser intensity of $7.5 \mathrm{~W} \mathrm{~cm}^{-2}$. We observed a wide band centred at $584 \mathrm{~nm}(2.123 \mathrm{eV})$. The PL band has a FWHM of about $0.122 \mathrm{eV}$ and an asymmetrical Gaussian lineshape. These features are typical of emission bands due to donor-acceptor pair transitions observed in ternary compounds [10]. We also note that the peak energy position and the emission band intensity change with temperature.

Figure 2 presents the shift of the peak energy position towards lower energies with increasing temperature. The variation of the direct band gap energy of TlInSeS as a function of temperature [1] is also indicated for comparison in this figure. Since the temperature coefficient of the band gap energy of the TIInSeS crystals is negative (i.e. $\mathrm{d} E_{g} / \mathrm{d} T=-1.35 \times 10^{-4}[1]$ ), the peak energy due to the donor-acceptor transition should decrease with band gap energy as the temperature increases [11]. The observed shift of the peak energy position towards lower energies satisfies the temperature dependence expected for the transitions involving donor and acceptor levels bound with respective band edges.

In donor-acceptor pair recombination, photoexcited carriers trapped at ionized donors and acceptors recombine radiatively. For the emission energy of the donor-acceptor pair, we recall a general expression of the form [12]

$$
h v=E_{g}-E_{a}-E_{d}+e^{2} /(\varepsilon R),
$$

where $E_{g}$ is the band gap energy, $E_{d}$ and $E_{a}$ are the donor and acceptor binding energies, $R$ is the distance between pairs and $\varepsilon$ is the static dielectric constant. The fourth term in the 


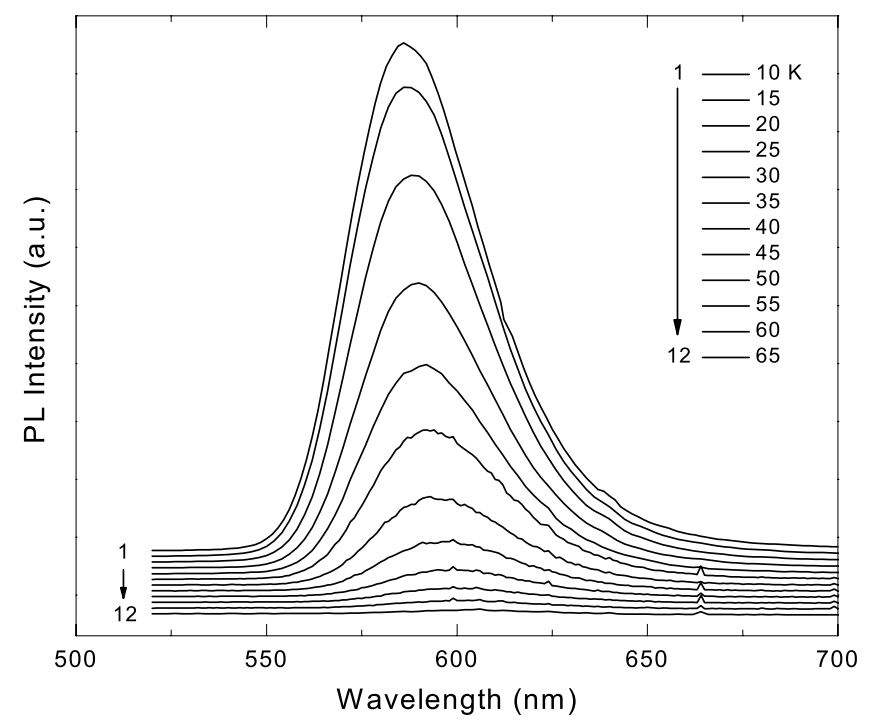

Figure 1. PL spectra of TIInSeS crystal in the temperature range 10-65 K. Excitation laser intensity $L=7.5 \mathrm{~W} \mathrm{~cm}^{-2}$.

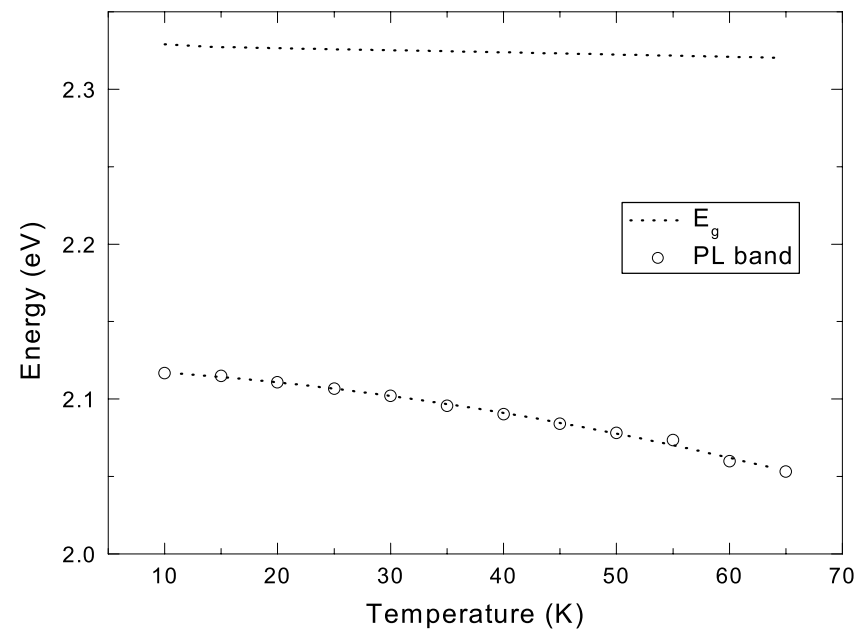

Figure 2. Temperature dependence of the emission band peak energy for TIInSeS. Temperature dependence of the direct band gap energy is also plotted for comparison. The dotted curves are only guides for the eye.

expression is a correction for Coulomb interaction between pairs and may be effective only at short distances. At low temperatures photoexcited charge carriers are bound to donors and acceptors and recombine with closest neighbours. Due to thermal emission of carriers bound to acceptor and donor levels one expects an increase in the pair separation $R$ with increasing temperature and the pairs that are apart also play a role in the recombination process. As equation (1) makes clear, the contribution from the distant pairs results in a red shift of the peak energy of the bands.

From figure 1 it can be seen that the intensity of the band decreases with respect to increasing temperature. A rapid thermal quenching of the PL band is observed above $T=35 \mathrm{~K}$ 


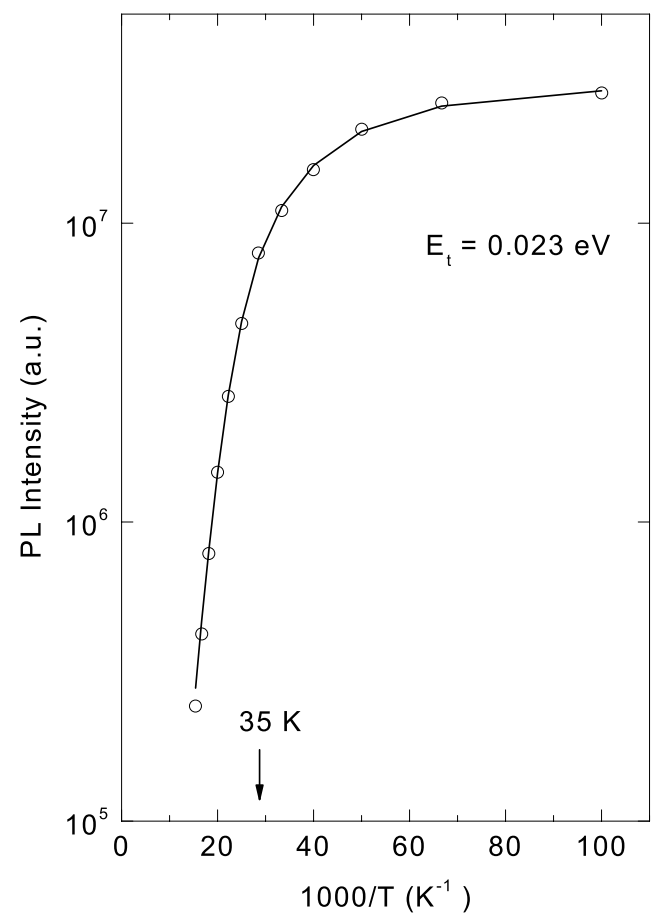

Figure 3. Temperature dependence of integrated PL band intensity for TIInSeS. The arrow at $35 \mathrm{~K}$ shows the starting point of the intensive quenching process.

(figure 3). The experimental data for the temperature dependence of the integrated PL band intensity can be fitted by the following expression [13]:

$$
I(T)=\frac{I_{0}}{1+\alpha_{1} T^{3 / 2}+\alpha_{2} T^{3 / 2} \exp \left(-E_{t} / k T\right)}
$$

where $I_{0}$ is a proportionality constant, $E_{t}$ is the thermal activation energy, $k$ is the Boltzmann constant and $\alpha_{1}$ and $\alpha_{2}$ are the fitting parameters associated with the temperature dependence of the capture cross sections of the donor and acceptor impurity levels. Figure 3 shows the temperature dependence of the integrated intensity as a function of the reciprocal temperature in the 10-65 K range. The best fit using equation (1), demonstrated by the full curve in figure 3 , has been achieved with parameters $I_{0}=29.3 \times 10^{6}, E_{t}=0.023 \mathrm{eV}, \alpha_{1}=1.1 \times 10^{-4}$ and $\alpha_{2}=3.6 \times 10^{-2}$. Since the TIInSeS crystal is a p-type semiconductor, we consider that the impurity level is an acceptor level located at $0.023 \mathrm{eV}$ above the top of the valence band. A shallow acceptor level in undoped TIInSeS crystal may be associated with the presence of uncontrolled impurities or defects.

Figure 4 presents the PL spectra for 16 different laser intensities at $T=10 \mathrm{~K}$. A careful inspection of the data shows that the emission band maximum slightly shifts towards higher energies $\left(\Delta E_{p}=52 \mathrm{meV}\right)$ with increasing excitation laser intensities from 0.023 to $7.5 \mathrm{~W} \mathrm{~cm}^{-2}$ (i.e. $21 \mathrm{meV}$ per decade of intensity of exciting radiation). The observed blue shift is characteristic for the donor-acceptor pair recombination spectra $[11,12]$. The magnitude of the observed blue shift is typical of ternary and quaternary chalcogenide compounds such as $\mathrm{AgGaS}_{2}$ [10], $\mathrm{Tl}_{2} \mathrm{InGaS}_{4}$ [14], $\mathrm{TlInS}_{2}$ [15], $\mathrm{HgInGaS}_{4}$ [16] and $\mathrm{CuIn}_{1-x} \mathrm{Ga}_{x} \mathrm{Se}_{2}$ [17], which are $64,40,20,20$ and $15 \mathrm{meV}$ per decade of intensity of exciting radiation, respectively. 


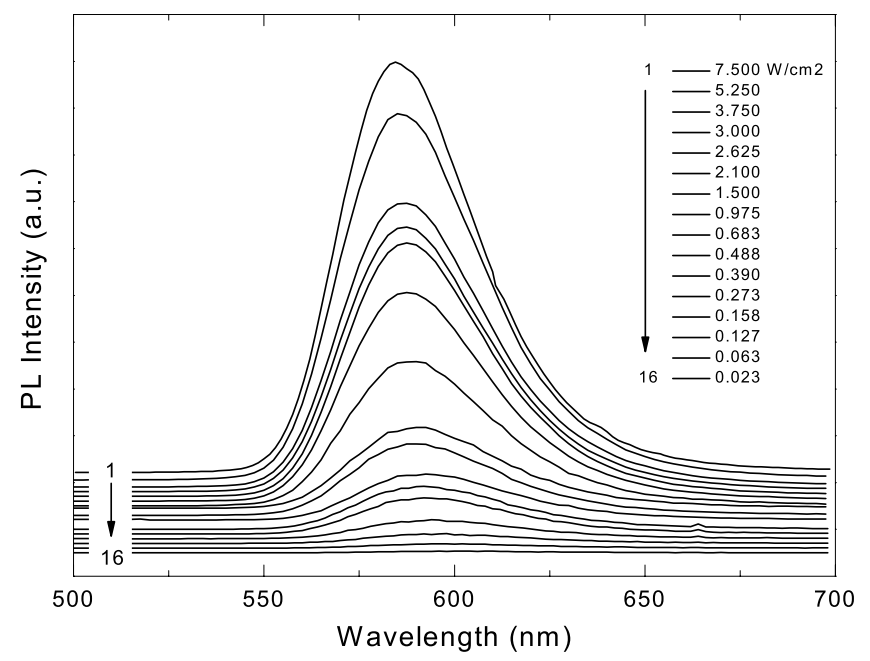

Figure 4. PL spectra of TIInSeS as a function of excitation laser intensity at $T=10 \mathrm{~K}$.

At low excitation laser intensities only a small fraction of the donor and acceptor levels trap carriers. This leads to recombination from distant pairs only. At high enough excitation laser intensities all donors and acceptors are excited which leads to a contribution from closer pairs as well. The energy of the emitted photon during a donor-acceptor pair transition has a positive contribution from a Coulombic interaction between ionized impurities. This contribution increases as the separation between the pairs decreases [12]. Furthermore, radiative transition probabilities for different pair separations are different and decrease exponentially as a function of the pair distance [12]. Distant pair recombination (contributing to the low energy part of a donor-acceptor pair emission band) saturates at high excitation laser intensities, whereas close pairs have a larger transition probability and can accommodate more carriers. We therefore observe a shift of the emission band peak energy to higher energy as the excitation laser intensity increases.

The dependence of the emission band peak energy $\left(E_{p}\right)$ at $10 \mathrm{~K}$ as a function of excitation laser intensity $(L)$ is given in figure 5 . The experimental data in figure 5 is then fitted by the following expression:

$$
L\left(E_{p}\right)=L_{0} \frac{\left(E_{p}-E_{\infty}\right)^{3}}{\left(E_{B}+E_{\infty}-2 E_{p}\right)} \exp \left[-\frac{2\left(E_{B}-E_{\infty}\right)}{E_{p}-E_{\infty}}\right]
$$

where $L_{0}$ is a proportionality constant, $E_{B}$ is the emitted photon energy of a close donoracceptor pair separated by a shallow impurity Bohr radius $\left(R_{B}\right)$ and $E_{\infty}$ is the emitted photon energy of an infinitely distant donor-acceptor pair [18]. From a nonlinear least squares fit to the experimental data, the photon energy values for an infinitely distant donor-acceptor pair and a close donor-acceptor pair separated by $R_{B}$ are found to be $E_{\infty}=2.063 \mathrm{eV}$ and $E_{B}=2.168 \mathrm{eV}$, respectively. These limiting photon energy values are in good agreement with the band gap energy $\left(E_{g}=2.328 \mathrm{eV}\right)$ and the observed values of the peak energy position (i.e. $E_{\infty}<2.070 \mathrm{eV}<E_{P}<2.122 \mathrm{eV}<E_{B}<E_{g}$ ) at $10 \mathrm{~K}$.

We have also studied the variation of the integrated emission band intensity versus the excitation laser intensity. The experimental data can be fitted by the simple power law

$$
I \propto L^{\gamma}
$$

where $I$ is the PL intensity, $L$ is the excitation laser intensity and $\gamma$ is a dimensionless exponent. 


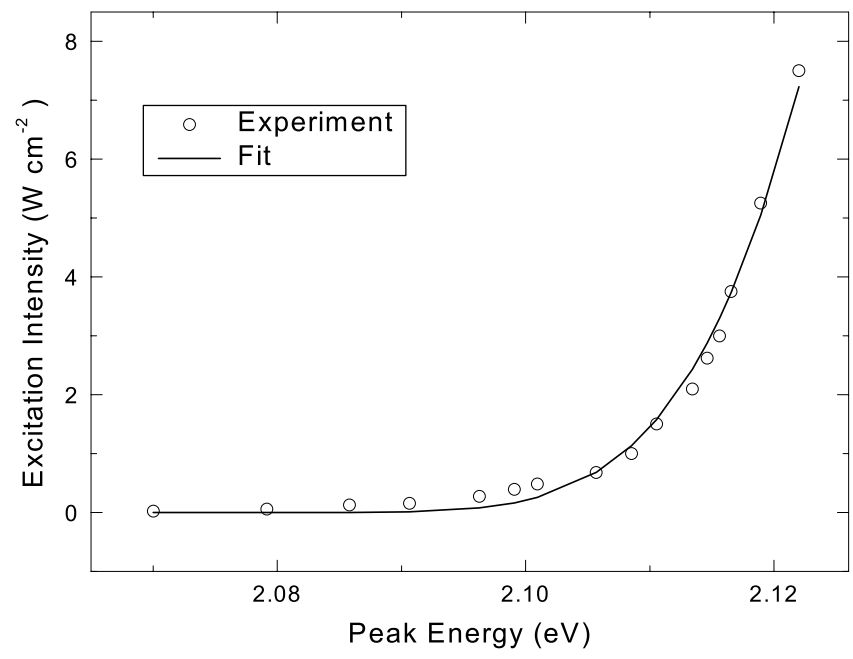

Figure 5. Excitation laser intensity versus emission band peak energy at $T=10 \mathrm{~K}$. The full curve represents the theoretical fit using equation (3).

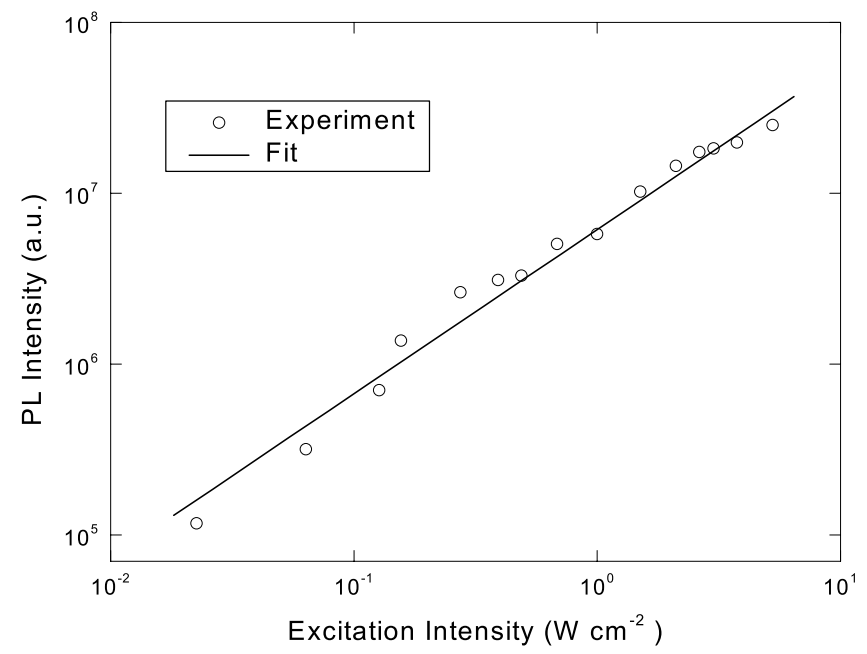

Figure 6. Dependence of integrated PL band intensity on excitation laser intensity at $T=10 \mathrm{~K}$. The full curve gives the theoretical fit using equation (4).

It was found that the integrated PL band intensity increases sublinearly (i.e. $\gamma=0.96$ ) with respect to the excitation intensity (figure 6). For an excitation laser photon with an energy exceeding the band gap energy $E_{g}$, the coefficient $\gamma$ is generally $1<\gamma<2$ for the free- and bound-exciton emission, and $\gamma \leqslant 1$ for free-to-bound and donor-acceptor pair recombinations [19]. Thus, the obtained value of $\gamma=0.96$ confirms our assignment of the observed emission band in TIInSeS spectra to donor-acceptor pair recombination.

The analysis of the PL spectra as a function of temperature and excitation laser intensity allows one to obtain a possible scheme for the donor-acceptor levels located in the forbidden energy gap of the TIInSeS crystal. These donor-acceptor levels are involved in the radiative recombination of the photoexcited carriers observed in this work. In the proposed scheme, 
shallow acceptor level $\boldsymbol{a}$ is located at $0.023 \mathrm{eV}$ above the top of the valence band. On the basis of the general expression for the emission energy of a donor-acceptor pair [12] and taking into account $E_{g}$ and $E_{\infty}$, the sum of the activation energies of the donor and acceptor levels, involved in PL band emission, has been estimated as being

$$
E_{d}+E_{a}=E_{g}-E_{\infty}=2.328-2.063 \mathrm{eV}=0.265 \mathrm{eV}
$$

Consequently, a donor level $\boldsymbol{d}$, located at $0.242 \mathrm{eV}$ below the bottom of the conduction band, is introduced into the forbidden energy gap of TIInSeS. We propose that the moderately deep donor level in a p-TIInSeS layered crystal is associated with dislocations or stacking faults, which are easy to produce because of the weakness of the van der Waals forces between the layers. Unintentional impurities may also be involved. Similarly, a moderately deep donor level $\left(E_{d}=0.25 \mathrm{eV}\right)$ was also revealed in the p-TIInS 2 layered crystal [15].

Taking into account the above considerations, the observed emission band in the PL spectra has been attributed to the radiative recombination of an electron occupying a donor level $\boldsymbol{d}\left(E_{d}=0.242 \mathrm{eV}\right)$ and a hole occupying an acceptor level $\boldsymbol{a}\left(E_{A}=0.023 \mathrm{eV}\right)$.

It is worth noting the absence of the so-called free-to-bound emission features in the PL spectra within the quenching temperature region. After acceptor ionization, the resulting free holes may be captured by the same donor level which is a member of a donor-acceptor pair, and as a result a new PL band should appear at higher energy. In our samples we observed only one stage of thermal quenching and no PL band appears at the higher energy with increasing temperature (figure 1). Indeed, in the case of a deep donor level, the temperature quenching usually shows only one stage, because the deep donor-to-valence band recombination has a fairly small probability [20].

\section{Conclusions}

The results obtained from the PL study of TIInSeS single crystals in the 460-800 nm wavelength and in the 10-65 K temperature range show that the PL intensity of the emission band decreases with increasing temperature. A rapid thermal quenching of the emission band is observed above $T=35 \mathrm{~K}$. This behaviour was understood by introducing a shallow acceptor level located at $0.023 \mathrm{eV}$ above the valence band and a moderately deep donor level located at $0.242 \mathrm{eV}$ below the conduction band in the TllnSeS crystal. The blue shift of the emission band peak energy with increasing excitation laser intensity is explained using the inhomogeneously spaced donoracceptor pair model. Also, the integrated intensity of the emission band increases sublinearly with respect to the excitation laser intensity and confirms our assignment that the observed emission band in TIInSeS is due to donor-acceptor pair recombination.

\section{References}

[1] Akhmedov A M, Bakhyshov A E, Lebedev A A and Yakobson M A 1978 Sov. Phys.-Semicond. 12299

[2] Hanias M, Anagnostopoulos K, Kambas K and Spyridelis J 1989 Physica B 160154

[3] Kalkan N, Hanias M P and Anagnostopoulos A N 1992 Mater. Res. Bull. 271329

[4] Kerimova E, Mustafaeva S, Guseinova D, Efendieva I, Babaev S, Mamedov T G, Mamedov T S, Salaeva Z and Allakhverdiev K 2000 Phys. Status Solidi a 179199

[5] Kalomiros J A and Anagnostopoulos A N 1994 Phys. Rev. B 507488

[6] Gasanly N M, Dzhavadov B M, Tagirov V I and Vinogradov E A 1979 Phys. Status Solidi b 95 K27

[7] Bakhyshov N A, Gasanly N M, Dzhavadov B M, Tagirov V I and Efendiev S M 1979 Phys. Status Solidi b 91 K1

[8] Aydinli A, Gasanly N M, Yilmaz I and Serpenguzel A 1998 Semicond. Sci. Technol. 14599

[9] Gasanly N M, Ozkan H and Culfaz A 1995 Cryst. Res. Technol. 30109

[10] Leyris J P, Aicardi J P and Soule S 1983 J. Lumin. 2865 
[11] Pankove J I 1971 Optical Processes in Semiconductors (Englewood Cliffs, NJ: Prentice-Hall) p 150

[12] Yu P Y and Cardona M 1995 Fundamentals of Semiconductors (Berlin: Springer) p 348

[13] Krustok J, Collan H and Hjelt K 1997 J. Appl. Phys. 811442

[14] Gasanly N M, Serpengüzel A, Gürlü O, Aydinli A and Yilmaz I 1998 Solid State Commun. 108525

[15] Aydinli A, Gasanly N M, Yilmaz I and Serpengüzel A 1999 Semicond. Sci. Technol. 14599

[16] Anedda A, Casu M B, Serpi A, Barlakov I I, Tiginyanu I M and Ursaki V V 1997 J. Phys. Chem. Solids 58325

[17] Bacewicz R, Dzierzega A and Trykozko R 1993 Japan. J. Appl. Phys. 32 (Suppl. 3) 3194

[18] Zacks E and Halperin A 1972 Phys. Rev. B 63072

[19] Schmidt T, Lischka K and Zulehner W 1992 Phys. Rev. B 458989

[20] Krustok J, Schon J H, Collan H, Yakushev M, Madasson J and Bucher E 1999 J. Appl. Phys. 86364 